Supporting Information for

\title{
Prediction of a Feshbach Resonance in the Below-the-Barrier Reactive Scattering of Vibrationally Excited HD with $\mathrm{H}$
}

Boyi Zhou, ${ }^{\dagger .}$ Benhui Yang, ${ }^{\dagger}$ N. Balakrishnan, ${ }^{\S}$ B. K. Kendrick, ${ }^{\|}$and P. C. Stancil ${ }^{*}{ }^{\dagger}$

${ }^{\dagger}$ Department of Physics and Astronomy and Center for Simulational Physics,

University of Georgia, Athens, Georgia 30602, United States

${ }^{\ddagger}$ Key Laboratory of Materials Modification by Laser, Electron, and lon Beams

(Ministry of Education), School of Physics, Dalian University of Technology, Dalian 116024, P. R. China

${ }^{\S}$ Department of Chemistry and Biochemistry, University of Nevada, Las Vegas,

Nevada 89154, United States

"Theoretical Division (T-1, MS B221), Los Alamos National Laboratory, Los Alamos, New Mexico 87545, United States

*Corresponding author. Email: pstancil@uga.edu 


\begin{tabular}{|c|c|c|c|}
\hline & $\mathrm{E} \leqslant 1000 \mathrm{~cm}^{-1}$ & $\mathrm{E}>1000 \mathrm{~cm}^{-1}$ & \\
\hline jtot & $0-30$ & $0-120$ & Total angular momentum quantum number $J$ \\
\hline ipar & $(-1)^{J}$ & $(-1)^{J}$ & Triatomic parity eigenvalue $P$ \\
\hline jpar & 0 & 0 & Diatomic parity eigenvalue $p$ \\
\hline $\operatorname{rmax}$ & 20 & 15 & Maximum hyperradius $\rho_{\max }$ (in bohr) \\
\hline$m t r$ & 5000 & 200 & Number of log derivative propagation sectors \\
\hline jmax & 15 & 15 & Maximum rotational quantum number of any channel \\
\hline kmax & 10 & 10 & Helicity truncation parameter $k_{\max }$ \\
\hline emax & 2.7 & 2.7 & Maximum internal energy in any channel (in eV) \\
\hline
\end{tabular}

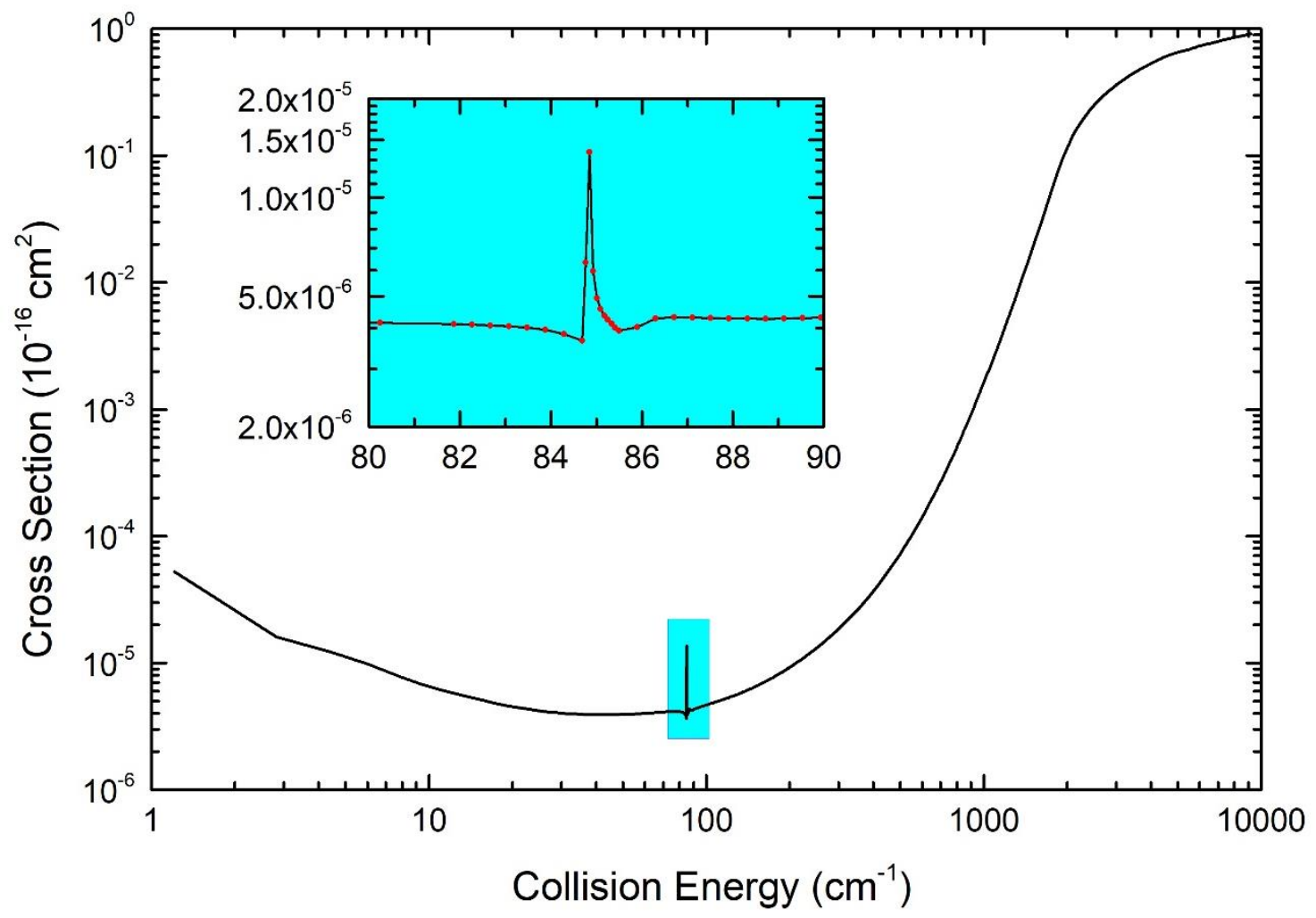

Figure S1. Collision energy variation of the cross section for the reactive process (sum over all final states) for the $\operatorname{HD}(v=1, j=0)+\mathrm{H}$ reaction. 

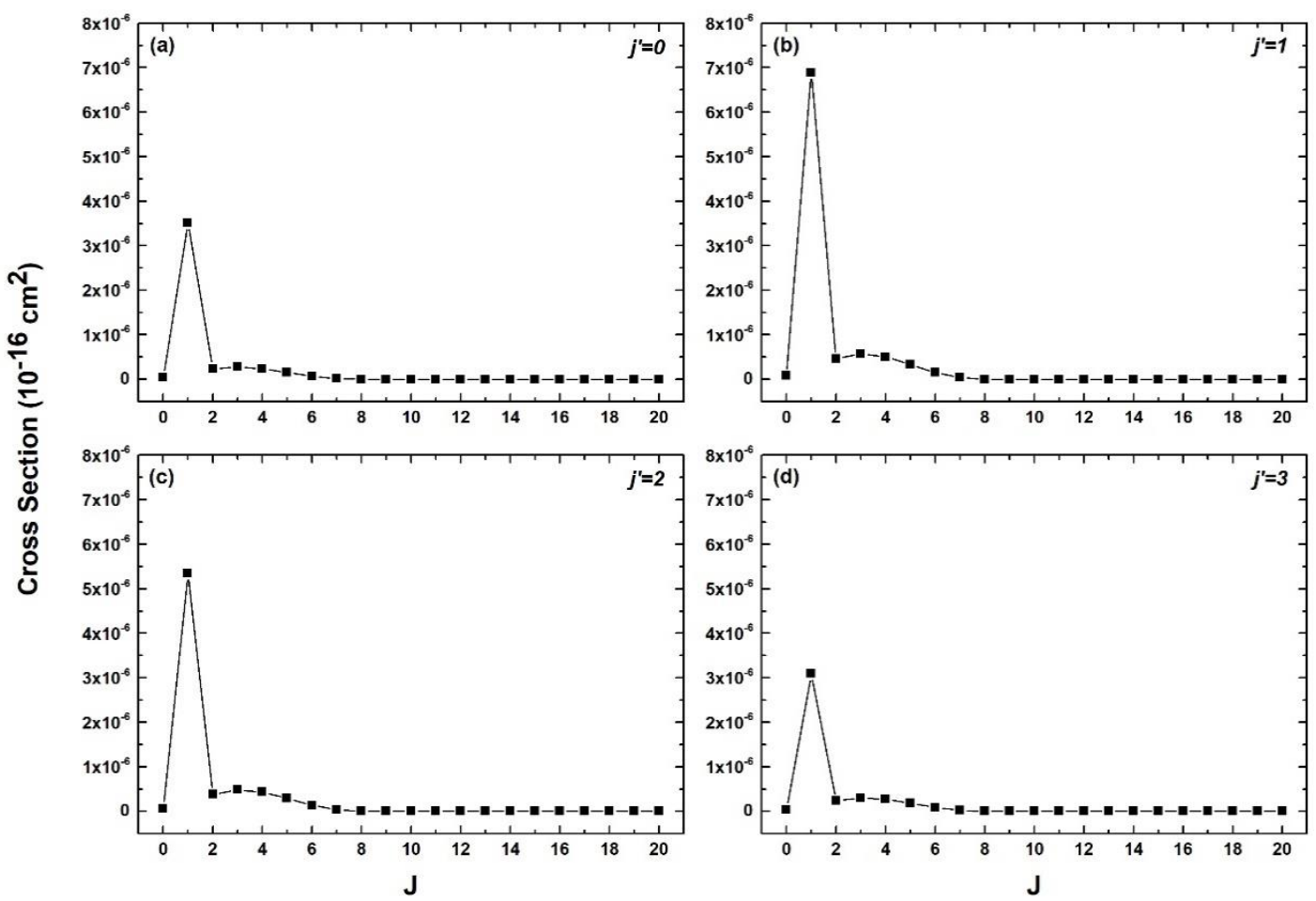

Figure S2. Contributions from each $J$ to the cross sections for the $\operatorname{HD}(v=1, j=0)+\mathrm{H} \rightarrow \mathrm{D}+$ $\mathrm{H}_{2}\left(v^{\prime}=0, j^{\prime}\right)$ reaction with rotational final states (a) $j^{\prime}=0,(\mathrm{~b}) j^{\prime}=1$, (c) $j^{\prime}=2$, (d) $j^{\prime}=3$.

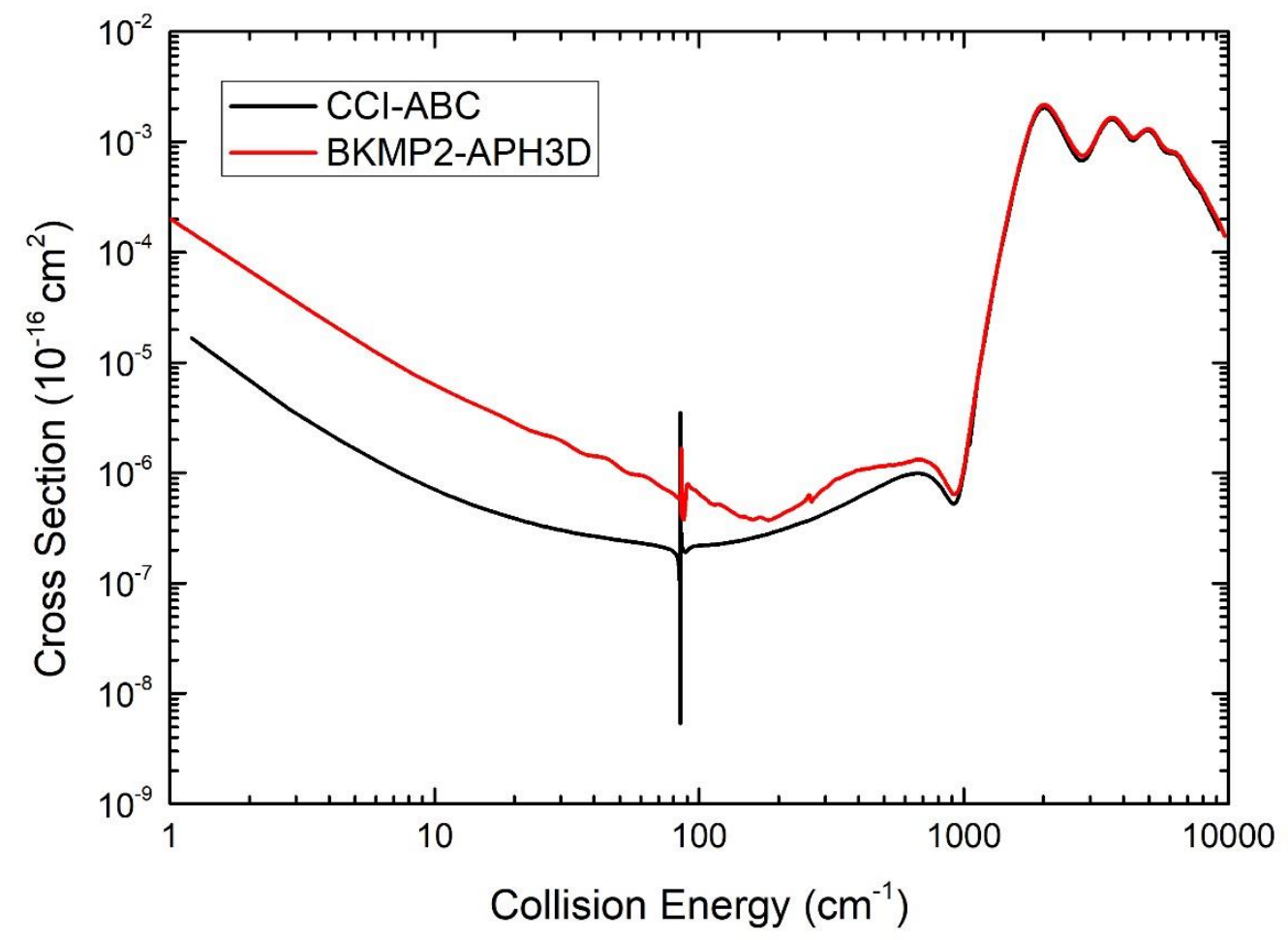

Figure S3. A comparison of collision energy variation of the cross section for $\operatorname{HD}(v=1, j=0)+$ $\mathrm{H} \rightarrow \mathrm{D}+\mathrm{H}_{2}\left(v^{\prime}=0, j^{\prime}=0\right)$ reaction on the CCI PES using the $\mathrm{ABC}$ program (black line) and on the BKMP2 PES using the APH3D program (red line) for $J=1$. 


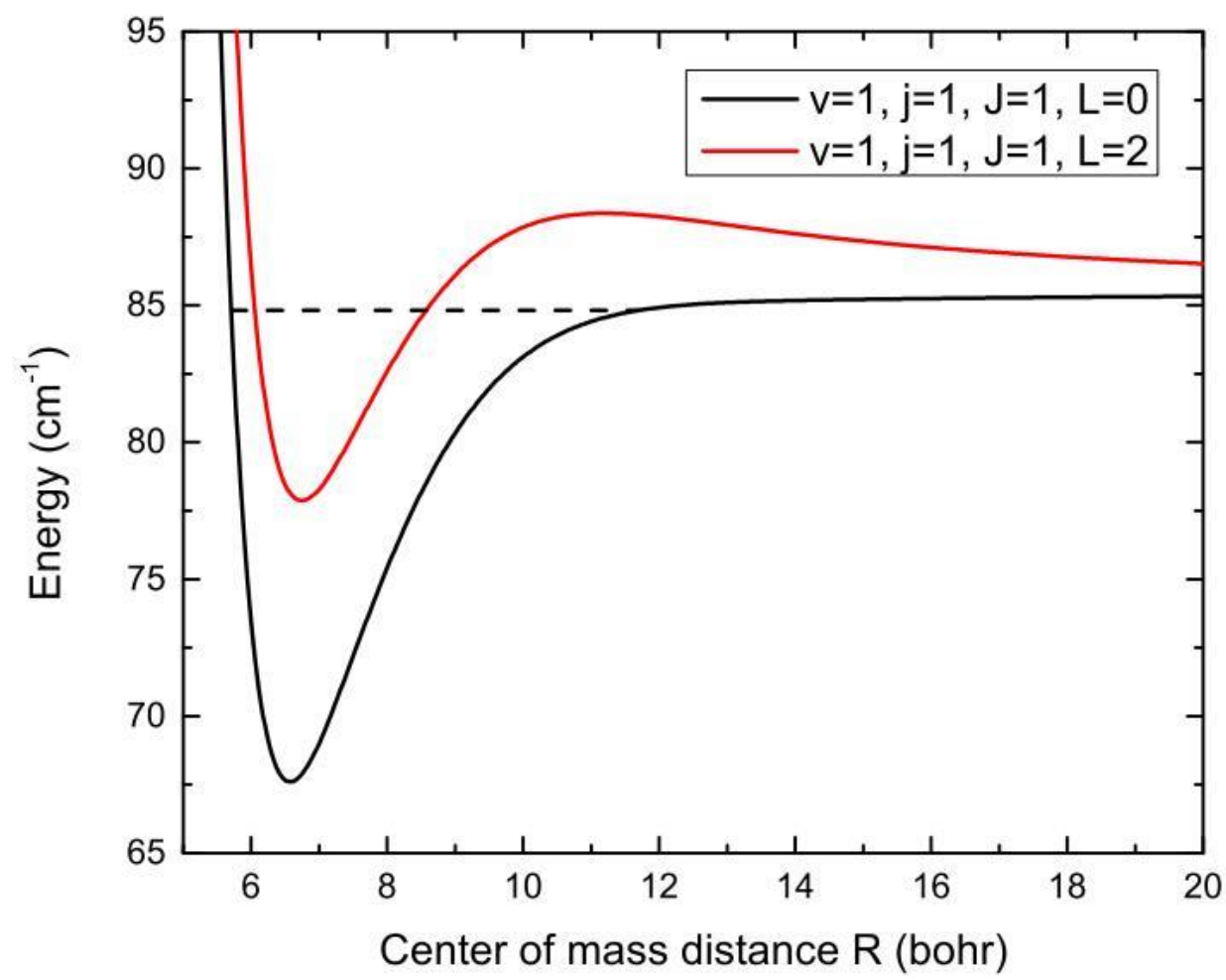

Figure S4. Adiabatic potentials correlating with different $(v, j)$ states of $\operatorname{HD}$ in $\operatorname{HD}(v=1, j=0)$ collisions for $J=1$ on the BKMP2 PES.

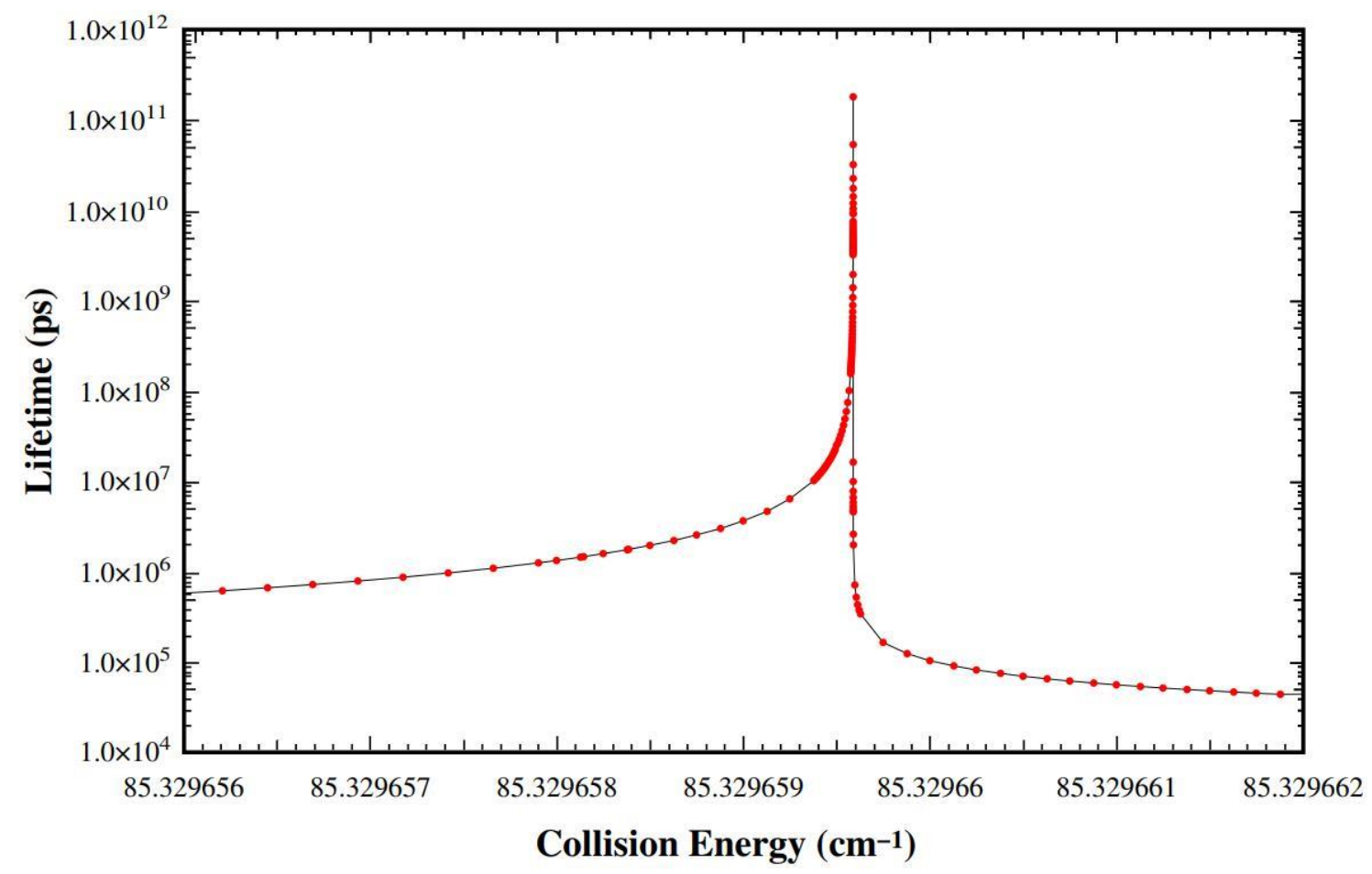

Figure S5. Lifetime of the Feshbach resonance in the below-the-barrier reactive scattering of the HD $(v=1, j=0)+\mathrm{H}$ collision on the BKMP2 PES using the APH3D program for $J=1$. 


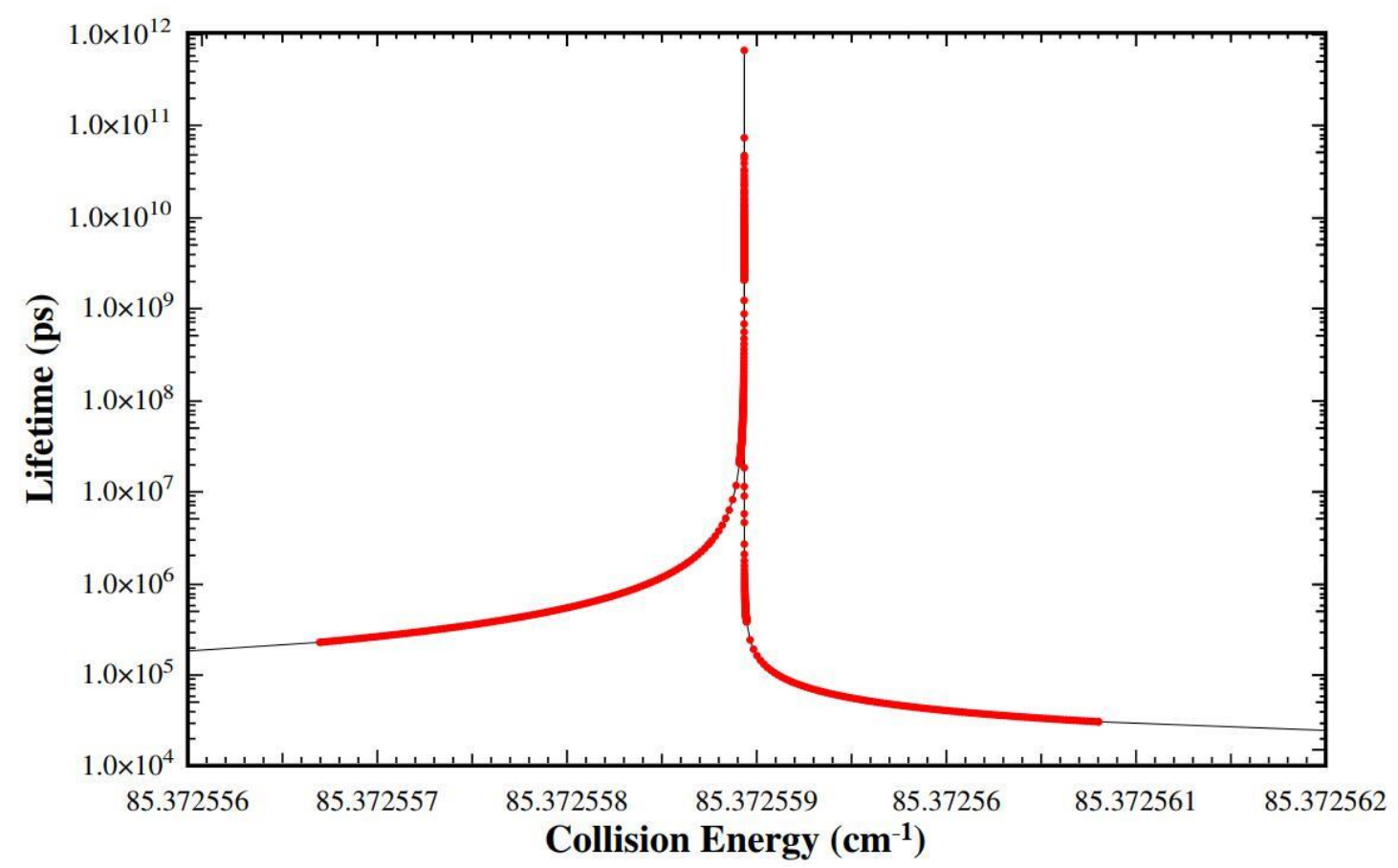

Figure S6. Lifetime of the Feshbach resonance in the below-the-barrier reactive scattering of the HD $(v=1, j=0)+\mathrm{H}$ collision on the CCI PES using the APH3D program for $J=1$. 